\title{
ESTADO EMOCIONAL Y CONDUCTA DE ENFERMEDAD EN PACIENTES CON VIH/SIDA Y ENFERMOS ONCOLÓGICOS
}

\author{
M. TERESA EDO y RAFAEL BALLESTER \\ Universidad Jaime I de Castellón
}

\begin{abstract}
Resumen: El objetivo del presente trabajo es ofrecer datos relativos al estado emocional y la conducta de enfermedad de personas con infección por VIH en comparación con pacientes oncológicos y población general. La muestra utilizada es de 180 sujetos. Los instrumentos aplicados evalúan: ansiedad rasgo y estado, depresión, autoestima, conducta anormal de enfermedad, percepción de apoyo social y grado de interferencia de la enfermedad. Los resultados indican que los pacientes seropositivos muestran un perfil psicológico con mayor grado de ansiedad estado y rasgo y niveles de depresión significativamente más altos que los otros grupos, así como una autoestima significativamente menor. Asimismo, los pacientes con infección por VIH manifiestan mayor preocupación por su salud, percepción de apoyo social significativamente menor que la de los pacientes oncológicos y también mayor grado de interferencia de la enfermedad en sus vidas. Se concluye la necesidad de un abordaje terapéutico específico para los pacientes con VIH que tenga en consideración su perfil diferencial respecto a otras enfermedades consideradas como graves.
\end{abstract}

Palabras clave: Conducta de enfermedad, estado emocional, infección por VIH, cáncer.

\section{Emotional distress and illness behavior in HIV/AIDS and cancer patients}

\begin{abstract}
The aim of this paper is to present data as to emotional distress and illness behavior of HIV patients when compared to cancer patients and the general population. The sample consisted of 180 subjects. Questionnaires were used to evaluate: trait anxiety, state anxiety, depression, selfesteem, abnormal illness behavior, perceived social support and interference through illness. The results indicate that HIV patients have a psychological profile with higher trait and state anxiety, significantly higher levels of depression, and significantly lower self-esteem than the other groups. Furthermore, HIV patients worry more about their health, perceive significantly less social support than cancer patients and a higher interference from the illness in their lives. Our conclusion points at the need for a specific therapeutic approach for HIV patients that takes into consideration their differential profile when compared to other major diseases.
\end{abstract}

Keywords: Illness behavior, emotional distress, HIV infection, cancer.

\section{INTRODUCCIÓN}

Muchas personas a las que se les diagnostica el VIH, especialmente por el estigma asociado a esta enfermedad, reciben el diagnóstico como una sentencia de muerte que, cuanto menos, les ocasiona importantes desequilibrios en su vida personal. Los cambios físicos y psicológicos producidos por la infección del VIH y su trata-

Recibido 23 mayo 2006; aceptado 27 julio 2006.

Correspondencia: Rafael Ballester, Dpto. Psicologia Básica, Clínica y Psicobiología, Universidad Jaime I de Castellón, Avda. Sos Baynat, 12071 Castellón (España). Correo-e: rballest@pab.uji.es miento afectan severamente a la calidad de vida de los pacientes debido a la idiosincrasia de esta enfermedad (Davis, 2004). Recordemos que el SIDA es una enfermedad calificada actualmente como crónica y, por tanto, incurable, con las implicaciones de ajuste personal y emocional que ello implica (visitas y controles medicos, tratamiento indefinido, complejo y con efectos secundarios, aparición de enfermedades oportunistas...). Además, el padecer una enfermedad transmisible y asintomática durante un período de tiempo, no sólo supone una gran limitación y fuente permanente de malestar psicológico para las personas afectadas que, sin sentirse enfermas, han de seguir determinadas pautas 
médicas y adoptar una serie de medidas preventivas para evitar infectar a otros o reinfectarse sino que, además implica un cuestionamiento del propio futuro y proyecto de vida.

Las reacciones emocionales que experimentan las personas afectadas por el VIH pueden calificarse como alteraciones universales, generales, lógicas y variables. Universales en cuanto pueden ser experimentadas, en mayor 0 menor cantidad o intensidad, por los seropositivos o enfermos de SIDA de todas las partes del mundo; generales y no especificas o exclusivas de la infección por el VIH porque ya han sido descritas con anterioridad en otros tipos de enfermedades; son consecuencias lógicas de la enfermedad puesto que se trata de respuestas de la persona ante un suceso estresante que pone en peligro su existencia; y variables debido a que existen diferentes reacciones entre los afectados y dentro del propio enfermo ya que, su estado psicológico fluctúa mucho a lo largo de todo el proceso de la enfermedad.

En la revisión de la literatura realizada por Ballester (1999) se señalan los siguientes aspectos psicológicos manifestados por los pacientes con VIH: baja autoestima, miedo a perder el atractivo físico e hipocondría (Morin, Charles y Malyon, 1984); negación, ira, aceptación, resignación y preparación para la muerte (Nichols, 1983) expresando una evolución similar a la que se produce en los enfermos terminales (Kim y Rickman, 1988; Levenson, 1988); somatización, síntomas obsesivo-compulsivos (Bruce y Stevens, 1992; Krikorian, Kay y Liang, 1995); reaparición de conflictos sobre la orientación homosexual y exacerbación de la homofobia (Ross y Rosser, 1988); disminución de la concentración y pérdida de memoria (Naber et al., 1989; King, 1989; Ayers, Abrams, Newell y Friedrich, 1989; Kermani, Borod, Brown y Tunnell, 1985); fobias especificas, trastomo de personalidad antisocial, deseo sexual hipoactivo (Green, 1994); abuso del alcohol (Pace, Brown, Rundell, Paolucci et al., 1990); problemas de sueño, desmoralización, uso excesivo de sedantes (Martin y Dean, 1993), consumo de drogas, etc.

En cualquier caso, parece que el primer cuadro clínico que experimenta la persona que acaba de conocer su seropositividad o desarrollo de la enfermedad es una reacción de estrés agudo asociada al VIH de carácter transitorio y que desaparece a los pocos días. Cuando las manifestaciones ante este shock emocional, descritas con anterioridad, se prolongan en el tiempo y/o son excesivamente intensas puede desencadenar un trastorno adaptativo dependiente de factores como: la respuesta de afrontamiento del afectado ante la enfermedad, su historia previa de patología mental, la actitud del entorno familiar, laboral y social y la disponibilidad de recursos de apoyo y asesoramiento. Aunque en este cuadro psicológico pueden aparecer síntomas depresivos (tristeza, sentimiento de desesperanza y desmoralización), ansiosos (miedos y preocupaciones) y quejas somáticas o alteraciones de conducta, éstos pueden remitir con consejo psicológico y apoyo emocional.

Parece que de todos los trastornos, los cuadros afectivos mixtos, con síntomas de ansiedad y depresión, son los trastornos psicológicos que con mayor frecuencia se describen en los pacientes con SIDA (Levenson, 1988; Weinrich, Atkinson, McCutchan y Grant, 1995; Remor, Carrobles, Arranz, Donate y Ulla, 2001; Morrison et al., 2002; Martin et al., 2002). Su presencia está asociada, a su vez, con factores psicosociales, como la disponibilidad o no de apoyo social, la propia percepción sobre la enfermedad, y el deterioro fisico provocado por la evolución de la infección (Bor, 1991). Una reacción ansiógena ante el VIH/SIDA, es esperable y prácticamente inevitable en todos los casos ya que, se trata de un mecanismo de adaptación de la persona a su nuevo contexto, la enfermedad. Si no se advierte, podemos pensar en la existencia de una negación psicológica de la enfermedad. La función de la ansiedad es la de preparar al afectado para que se mantenga alerta y activado ante las situaciones amenazantes que va a vivir en su proceso de enfermedad (exámenes médicos, hospitalizaciones, tratamiento farmacológico, notificación a los allegados, entablar relaciones con una persona de su agrado, etc).

Otros importantes cuadros clínicos que tienen como base la ansiedad y la depresión generada por la infección del VIH son las somatizaciones y la hipocondria (Ballester, 2005). Los pacientes seropositivos somatizan muchos síntomas o tienen la creencia de que están enfermos como consecuencia del malestar psicológico que conlleva saberse portador del VIH. La hipocondría relacionada con el SIDA no sólo la padecen los "worried well» portadores del VIH que permanecen asintomáticos sino, también la población general, las personas que practican comportamientos de riesgo y aquellas personas 
en contacto estrecho con los seropositivos (familiares, amigos y personal sanitario). Todos ellos, aunque por diferentes motivos, tienen preocupaciones y miedos en ocasiones excesivos, exagerados e irracionales sobre su salud que persisten a pesar de la información y explicaciones dadas por personal cualificado, las pruebas del VIH realizadas y los exámenes médicos efectuados. Es frecuente observar conductas de autoobservación e hipervigilancia sobre cualquier cambio en sus organismos que pueda ser interpretado como efecto de la infección. Por otra parte, García-Huete (1993) habla del papel que desempeñan las autoobservaciones «psicológicas» de los seropositivos asintomáticos. Éstas son las responsables de que los enfermos focalicen su atención en sus cogniciones, preocupándose por el tipo de pensamientos que tienen y dando lugar a síntomas de carácter ansioso o depresivo que se manifestarán a través de síntomas físicos los cuales, serán asociados a la infección. Se trata de un círculo vicioso sobre el que hay que intervenir.

Sin embargo, a pesar de que existe cierta literatura cientifica sobre los problemas psicológicos que suelen afectar a los pacientes con VIH, todavía sabemos poco acerca de sí en realidad podemos hablar de una problemática peculiar o especifica de estos enfermos, o bien los problemas que padecen no son muy distintos a los de pacientes con otras enfermedades graves como el cáncer. Paradójicamente, desde el punto de vista de la intervención psicológica de estos enfermos resulta fundamental conocer la especificidad o no de su perfil psicopatológico de cara a diseñar estrategias ajustadas a las particularidades de este perfil, aspecto que constituye el objetivo fundamental del presente trabajo.

\section{MÉTODO}

\section{Participantes}

La muestra del presente trabajo está compuesta por un total de 180 personas agrupadas en tres submuestras: el grupo VIH (G.VIH) formado por 63 pacientes seropositivos, asintomátiços, sintomáticos y enfermos de SIDA, de ambos sexos (71\% varones y $29 \%$ mujeres) con edades comprendidas entre los 23 y 52 años (media $=33, \mathrm{DT}=6,12)$ del Hospital General de Castellón (España); el grupo oncológico (G.ONC) con 57 pacientes, $58 \%$ varones y $42 \%$ mujeres, entre los 28 y 71 años (media $=55, \mathrm{DT}=10,21)$ que recibian tratamiento en el Hospital Provincial de Castellón por padecer diferentes tipos de cáncer; y el grupo control (G.C) compuesto por 60 sujetos sin ninguna de las enfermedades anteriores ni tampoco otras dolencias de importancia pero, con características semejantes, en cuanto al sexo ( $55 \%$ hombres y $45 \%$ mujeres) y la edad (media $=32, \mathrm{DT}=6,58$ ), a las del G.VIH. Con el fin de aseguramos que los participantes del grupo control no padecian ninguna enfermedad o dolencia fisica de importancia ni ningún problema psicológico que pudiera implicar un ánimo depresivo o ansioso se les preguntó por esta cuestión antes de la administración de los cuestionarios en entrevista individualizada.

\section{Variables Analizadas e Instrumentos}

Los resultados que expondremos en este trabajo forman parte de un amplio estudio en el que se explora el perfil psicosocial, el estado emocional y la conducta de enfermedad en pacientes con infección por VIH/SIDA españoles. Las variables clínicas que hemos considerado relevantes a tal efecto son: ansiedad rasgo y estado, depresión, autoestima, conducta anormal de enfermedad (hipocondría general, convicción de estar enfermo y preocupación por los síntomas, percepción psicológica frente a percepción somática de la enfermedad, inhibición y perturbación afectiva, negación de problemas cotidianos, irritabilidad y fobia a la enfermedad), percepción de apoyo social (a nivel general, en el trabajo, la familia, la pareja, los amigos, los médicos y el de otros enfermos) y grado de interferencia de la enfermedad en distintos ámbitos (laboral, social, familiar, sexual, de pareja, tiempo libre y a nivel global). Los cuestionarios administrados a los grupos que componen la muestra son los siguientes:

Inventario de Ansiedad Estado/Rasgo (STAI) (Spielberger, Gorsuch y Lushene, 1982). Consta de dos partes, con 20 items cada una de ellas. La primera $(A / E)$ evalúa un estado emocional 
transitorio, caracterizado por la presencia de sentimientos subjetivos, conscientemente percibidos, de atención y aprensión, así como de hiperactividad del sistema nervioso autónomo. La segunda (A/R) señala una propensión ansiosa, relativamente estable, que caracteriza a los individuos a percibir las situaciones como amenazantes. Los niveles de fiabilidad y validez son elevados (coeficiente de consistencia interna KR-20 de 0,92, 0,92, 0,93 y 0,90 y dos mitades de 0,94 A/E y 0,86 A/R) (Bermúdez, 1978).

Inventario de Depresión (BDI) de Beck y Steer (1993). Está compuesto por 21 ítems con contenido sintomatológico propio del trastorno depresivo. La clasificación de la intensidad de la depresión queda establecida, en función de los criterios normativos en: no deprimidos de 1 a 10 puntos, ligeramente deprimidos de 11 a 16 puntos, estados de depresión intermitentes de 17 a 20 puntos, moderadamente deprimidos de 21 a 30 puntos, depresión grave de 31 a 40 puntos y depresión extrema con más de $\mathbf{4 0}$ puntos. Se considera que una puntuación de 17 o más indica que la persona necesita ayuda profesional. La consistencia interna es de 0,86 y la fiabilidad test-retest de 0,85 (Ballesteros y Carrobles, 1989).

Cuestionario de Autoestima (RSEI) de Rosenberg (1979). Contiene 10 ítems que se puntúan con 4 alternativas de respuesta proporcionando información cualitativa y cuantitativa sobre el grado de satisfacción de una persona consigo misma. El punto de corte en la población adulta es de 29 (Ward, 1977). Los elevados niveles de validez y fiabilidad de esta escala (alpha de Cronbach de 0,87 y fiabilidad test-retest de 0,85 ) han sido bien documentados (Goldsmith, 1986; O'Brien, 1985).

Cuestionario de Conducta de Enfermedad (IBQ) de Pilowsky y Spence, (1983) (adaptación española de Ballester, 1992). Mide el patrón de conducta de enfermedad con sus 62 ítems de respuesta dicotómica (sí-no) formados en torno a 7 factores de primer orden y un indice de hipocondria (Byme y White, 1978; Pilowsky, Spence y Waddy, 1979; Zonderman, Heft y Costa, 1985). Los factores son los siguientes: hipocondría general (temor hacia la enfermedad y preocupacion excesiva por la salud), convicción de enfermedad (preocupación por los síntomas e interferencia en la vida normal), percepción psicológica vs somática de la enfermedad (una puntuación alta indica la creencia de que se padece problemas psicolo- gicos y que algunos síntomas físicos tienen un origen psicologico), inhibición afectiva, perturbación afectiva (tendencia a la depresión y ansiedad como consecuencia de la enfermedad), negación (tendencia a negar la enfermedad y los problemas cotidianos), irritabilidad (especialmente en situaciones interpersonales) y un índice Whiteley de Hipocondría (afirmación de la enfermedad y fobia a ésta). La fiabilidad test-retest es de 0,87 (Pilowsky y Spence, 1983).

Percepción del Apoyo Social en Infectados por VIH (PAS-VIH) de Ballester y Edo (Edo, Ballester y Pascual, 2003). Este instrumento mide, con sus 15 ítems de respuesta dicotómica (si-no), la percepción subjetiva de apoyo social recibido en el ámbito familiar, social, laboral, de pareja, amistades, equipo médico y otros enfermos. Y, también, proporciona información del nivel general de apoyo social percibido por parte de las personas infectadas por el VIH. Su fiabilidad evaluada a través de su consistencia interna (alfa de Cronbach) es de 0,86 .

Percepción del Apoyo Social en pacientes Oncologicos (PAS-ONC) de Ballester y Edo (Edo, Ballester y Pascual, 2003). Adaptación del cuestionario «Percepción del Apoyo Social en infectados por VIH (PAS-VIH)« para población oncológica. Permite obtener tanto la percepción del apoyo social recibido que tienen los pacientes con cáncer en las diferentes áreas de su vida (familiar, social, laboral, de pareja, amistades, equipo médico y enfermos) como el nivel general de apoyo social que ellos perciben. Su fiabilidad (consistencia interna a través de alfa de Cronbach) es de $\mathbf{0 , 8 3}$.

Escala de Adaptación de Echeburia y Corral (ampliada por Ballester y Edo, 1997) (Edo, 2003). Diseñada para evaluar el grado de interferencia a nivel laboral, social, de tiempo libre, relación de pareja, relación sexual, vida familiar y ámbito general, como consecuencia de la enfermedad.

\section{Procedimiento}

Todos los instrumentos de evaluación se aplicaron, de forma individual, tanto a los pacientes infectados por el VIH ingresados en la planta de Medicina Interna del Hospital General de Castellón (España) como, a los pacientes con cáncer de la Unidad de Oncología del Hospital Provincial 
de Castellon. Los enfermos, atendidos en sus propias habitaciones, firmaron una hoja de consentimiento en la que se les explicaba el objetivo del trabajo, la importancia de su colaboración y se les garantizaba el carácter anónimo y confidencial de todos los datos obtenidos.

Respecto a los participantes del grupo control, se les administró únicamente algunos de los cuestionarios, concretamente los que eran aplicables a una muestra general, ya que otros eran especificos para personas que sufrian una enfermedad orgánica como el cáncer o el SIDA y por lo tanto no eran adecuados ni habrían sido comprendidos por estos participantes. En el caso del cuestionario IBQ, dado que deseábamos comparar el perfil de nuestros pacientes con el de otros pacientes con problemas de ansiedad, utilizamos los datos de un trabajo previo de nuestro grupo de investigación, incluido el grupo control utilizado en ese estudio.

\section{Análisis estadísticos}

Para los análisis diferenciales entre los grupos de la muestra se han realizado pruebas t en el caso de variables continuas para dos grupos $\mathrm{y}$ análisis de varianza (Anovas) para las variables continuas con más de dos grupos con aplicación posterior de la prueba Scheffeé para establecer las diferencias entre los grupos. Los análisis se realizaron a través del paquete estadistico SPSS-PC+.

\section{RESULTADOS}

La ansiedad-estado como condición emocional transitoria de los seropositivos evaluados (ver Tabla 1) es superior a la de los pacientes oncológicos y los sujetos de la población general encontrándose diferencias significativas entre los tres grupos $(F=13,31, p<0,000)$. Las pruebas Scheffée muestran que se producen diferencias significativas entre la ansiedad estado del G.VIH con respecto al G.ONC ( $p<$ $0,001) \mathrm{y}$, también, con respecto al G.C ( $p<$ $0,001)$ sin que aparezcan dichas diferencias en la ansiedad entre el G.ONC y el G.C $(p<$ 0,511 ).

Con respecto a la ansiedad-rasgo los análisis de varianza realizados (Tabla 1) muestran la existencia de diferencias significativas entre los tres grupos $(F=31,94, p<0,000)$ siendo el G.VIH el que difiere significativamente en su nivel de ansiedad con respecto al G.ONC $(p<0,001)$ y al G.C $(p<0,001)$. Sin embargo, los estadísticos no muestran tampoco diferencias en esta variable entre los enfermos oncológicos y el G.C. $(p<$ 0,959).

Respecto a la depresión, los resultados expuestos en la Tabla 1, señalan a los pacientes infectados por el VIH como los más deprimidos. Sin embargo, la media del grupo sólo es indicativa de un estado de depresión intermedio, posiblemente, relacionado con situaciones puntuales de una enfermedad a la que temen y/o a circunstancias vitales. No obstante, sería conveniente seguir las recomendaciones ofrecidas en la interpretación del Inventario de Depresión de Beck, donde se aconseja la búsqueda de ayuda profesional para todas las personas con puntuaciones iguales o superiores a 17. En cuanto a las diferencias de depresión entre grupos, debemos comentar que los análisis estadísticos efectuados (Anovas y pruebas de Scheffée) nos muestran diferencias significativas $(F=42,19 ; p<0,001)$ entre todos y cada uno de los grupos, es decir, el G.VIH

Tabla 1. Diferencias entre los grupos de la muestra (ANOVA) en ansiedad depresión y autoestima

\begin{tabular}{|c|c|c|c|c|c|c|c|c|}
\hline & \multicolumn{2}{|c|}{$\begin{array}{c}\text { G.VIH } \\
(n=61)\end{array}$} & \multicolumn{2}{|c|}{$\begin{array}{l}\text { G.ONC } \\
(\mathrm{n}=57) \\
\end{array}$} & \multicolumn{2}{|c|}{$\begin{array}{c}\text { G.C } \\
(n=60)\end{array}$} & \multicolumn{2}{|c|}{. } \\
\hline & Media & DT & Media & DT & Media & DT & $F$ & Scheffee \\
\hline Ansiedad-E & 21,72 & 10,03 & 13,77 & 6,88 & 15,65 & 8,85 & $13,31^{* \ldots *}$ & VIH $>$ ONC y G.C \\
\hline Ansiedad- $\mathbf{R}$ & 30,39 & 10,49 & 17,64 & 8,59 & 18,17 & 9,92 & $31,94^{* n *}$ & VIH>ONC y G.C \\
\hline Depresión & 17,57 & 9,74 & 11,09 & 6,05 & 5,07 & 5,58 & $42,19 * * *$ & VIH $>$ ONC y G.C \\
\hline Autoestima & 28,85 & 4,54 & 33,20 & 3,37 & 32,31 & 4,69 & $14,37^{* * *}$ & G.VIH<ONC y G.C \\
\hline
\end{tabular}

$* * * p<0,001$ 
difiere con respecto al G.ONC y al G.C y, estos dos últimos grupos difieren significativamente entre sí.

En cuanto a la autoestima, nuestros pacientes seropositivos presentan una menor autoestima que las otras dos muestras de sujetos existiendo diferencias significativas entre la autoestima del G.VIH con el G.ONC y con el G.C $(F=14,37, p$ $<0,000$ ). Esto significa que, en general, las personas con VIH se sienten menos competentes para llevar a cabo actuaciones, experimentan más sentimientos de desaprobación hacia ellos mismos y se consideran menos merecedores de la aceptación de los demás que las personas enfermas de cáncer y la población general. Sin embargo, no se aprecian diferencias significativas en cuanto al grado de autoestima entre los enfermos oncológicos y el grupo control $(p<0,539)$ según indican las pruebas de Scheffée.

La siguiente variable estudiada es la Conducta Anormal de Enfermedad. En general, como puede observarse en la Tabla 2, los pacientes infectados por el VIH alcanzan puntuaciones medias superiores en todos los factores del I.B.Q a las obtenidas por los pacientes oncológicos excepto en el factor de Negación del Problema. Estos resultados parecen indicar que los pacientes con VIH comparados con los enfermos oncológicos tienen un perfil peculiar de conducta de enfermedad caracterizado por un mayor nivel de hipocondría $(t=4,79, p<$ $0,000)$, más problemas afectivos $(t=3,60, p<$ $0,001)$, menor tendencia a negar los problemas cotidianos y/o a explicarlos a partir de sus problemas físicos $(t=-5,91, p<0,000)$, un nivel más elevado de irritabilidad $(t=3,07, p<$ 0,003 ), así como, una elevada preocupación somática, afirmación de su enfermedad y fobia a ésta $(t=2,61, p<0,010)$. Asimismo los datos que exponemos en la Tabla 2 referentes a los resultados de un grupo control de personas sin enfermedades orgánicas ni problemas psicológicos diagnosticables, utilizado en un estudio previo (Ballester y Botella, 1993) nos muestran en general puntuaciones inferiores en el grupo control comparadas con las de nuestros grupos de pacientes.

Por lo que respecta a la percepción de apoyo social, en la Tabla 3, se observa cómo, en general, los enfermos con cáncer superan en puntuación media a los enfermos por VIH en el apoyo social percibido proveniente de ámbitos como el social $(t=-9,23, p<0,000)$, el laboral $(t=-$ $6,84, p<0,000)$, el familiar $(t=-5,75, p<$ $0,000)$, el círculo de amigos $(t=-4,49, p<0,000)$ y a nivel general $(t=-9,77, p<0,000)$. Probablemente, los enfermos del G.VIH entienden que revelar su condición de portador del VIH puede producirles consecuencias negativas por la estigmatización, las actitudes negativas o los conflictos que suscita el diagnóstico del VIH en la población general. Por ello, prefieren mantenerlo oculto $y$, consecuentemente, perciben menos apoyo que los otros enfermos. A pesar de estas circunstancias, los pacientes del G.VIH y G.ONC son comparables en cuanto al apoyo

Tabla 2. Resultados sobre el Perfil de Conducta Anormal de Enfermedad en los pacientes con VIH y con cáncer

\begin{tabular}{|c|c|c|c|c|c|c|c|c|}
\hline \multirow[b]{2}{*}{ Factores } & \multirow[b]{2}{*}{ IBQ } & \multicolumn{2}{|c|}{$\begin{array}{c}\text { G.VIH } \\
(n=45)\end{array}$} & \multicolumn{2}{|c|}{$\begin{array}{l}\text { G.ONC } \\
(n=57)\end{array}$} & \multirow{2}{*}{$\frac{\begin{array}{c}\text { Comparación } \\
\text { VIH/ONC }\end{array}}{t}$} & \multicolumn{2}{|c|}{$\begin{array}{c}\text { G.C } \\
(n=32)\end{array}$} \\
\hline & & Media & DT & Media & DT & & Media & DT \\
\hline & HG & 5,49 & 2,24 & 3,46 & 1,95 & $4,79 * * *$ & 1,9 & 2,3 \\
\hline & $\mathrm{CE}$ & 4,02 & 2,03 & 3,46 & 1,39 & 1,62 & 1,0 & 1,0 \\
\hline & PP & 2,38 & 1,81 & 1,72 & 1,69 & 1,86 & 2,2 & 0,8 \\
\hline & IA & 2,43 & 1,39 & 2,04 & 1,39 & 1,40 & 1,5 & 1,5 \\
\hline & PA & 3,50 & 1,68 & 2,28 & 1,66 & $3,60^{* * *}$ & 1,6 & 1,7 \\
\hline & NE & 2,86 & 1,77 & 4,44 & 0,77 & $-5,91 * * *$ & 2,1 & 1,5 \\
\hline & IR & 2,36 & 1,46 & 1,48 & 1,37 & $3,07^{* *}$ & 1,4 & 1,7 \\
\hline & IH & 8,70 & 2,84 & 7,25 & 2,62 & $2,61^{* *}$ & 3,0 & 2,9 \\
\hline
\end{tabular}

Nota: $\mathrm{HG}=$ Hipocondria General; CE = Convicción de estar Enfermo y Preocupación por los Síntomas; PP = Percepción Psicológica versus Somática de la Enfermedad; IA = Inhibición; PA = Perturbación Afectiva; NE = Negación de Problemas Cotidianos; IR = Irritabilidad o Enfado y Fobia a la Enfermedad; IH = Indice Whiteley de Hipocondria.

${ }^{* *} p<0,01, * * * p<0,001$. 
Tabla 3. Descripción de la muestra de pacientes con VIH y con cáncer según la percepción del apoyo social

\begin{tabular}{lccccc}
\hline & $\begin{array}{c}\text { G.VIH } \\
(\mathrm{n}=50)\end{array}$ & \multicolumn{3}{c}{$\begin{array}{c}\text { G.ONC } \\
(\mathrm{n}=57)\end{array}$} \\
\hline Media & DT & Media & DT & \multicolumn{1}{c}{} \\
\hline Social & 0,70 & 0,65 & 1,73 & 0,49 & $-9,23^{* * *}$ \\
Laboral & 0,72 & 0,76 & 1,59 & 0,53 & $-6,84^{* * *}$ \\
Familiar & 2,94 & 1,13 & 3,87 & 0,39 & $-5,75^{* * *}$ \\
Pareja & 0,85 & 0,36 & 0,96 & 0,20 & $-1,64$ \\
Amigos & 1,24 & 0,77 & 1,80 & 0,49 & $-4,49 * * *$ \\
Enfermos & 0,88 & 0,66 & 1,11 & 0,71 & $-1,71$ \\
Equipo médico & 2,12 & 0,98 & 2,31 & 0,66 & $-1,16$ \\
General & 8,44 & 2,39 & 12,38 & 1,71 & $-9,77^{* * *}$ \\
\hline
\end{tabular}

$* * p<0,001$.

percibido por otros enfermos en su misma situación, los médicos que los atienden y las parejas que los cuidan y quieren, o sea, quienes conocen su diagnóstico.

Para finalizar, como puede verse en la Tabla 4, el grado de interferencia de la enfermedad es mayor para los pacientes seropositivos que para los enfermos oncológicos en el ámbito social $(t=$ $2,66, p<0,009)$, familiar $(t=4,63, p<0,000)$ y de pareja $(t=3,55, p<0,001)$. Sin embargo, en el área laboral $(t=-2,19, p<0,030)$ son los pacientes con cáncer quienes consideran que su enfermedad interfiere en mayor medida que los pacientes con VIH, quienes suelen ocultar su estado serológico en su trabajo.

\section{CONCLUSIONES Y DISCUSIÓN}

Los resultados obtenidos nos evidencian que los pacientes con VIH comparados con los pacientes oncológicos y la población general muestran un perfil psicológico caracterizado por un mayor grado de ansiedad estado y rasgo, con niveles mayores de depresión, menor autoestima, una conducta de enfermedad marcada por una mayor perturbación afectiva, hipocondría, fobia la enfermedad, irritabilidad y preocupación excesiva por su salud, un menor apoyo social percibido en las áreas social, laboral, familiar, de amistades y a nivel general y una mayor interferencia de la enfermedad en el ámbito social, familiar y de pareja.

Podemos decir que, nuestros resultados apoyan en unos casos y contradicen en otros casos los obtenidos en otros estudios aunque algunas de las divergencias podrian entenderse por las características de las muestras (sexo, via de transmisión, estadio clínico, tamaño de la muestra etc.) e instrumentos de evaluación utilizados en los distintos estudios.

Así, según Hunter (1993), el estrés psicológico producido por el VIH/SIDA ocasiona diferentes

Tabla 4. Descripción de las muestras de pacientes en función del grado de interferencia de la enfermedad

\begin{tabular}{lccccc}
\hline & $\begin{array}{c}\text { G.VIH } \\
(\mathbf{n}=47)\end{array}$ & \multicolumn{2}{c}{$\begin{array}{c}\text { G.ONC } \\
(\mathbf{n}=57)\end{array}$} \\
\hline & Media & DT & Media & DT & t \\
Laboral & 2,62 & 1,94 & 3,40 & 1,66 & $-2,19 *$ \\
Social & 1,30 & 1,63 & 0,54 & 1,23 & $2,66 * *$ \\
Tiempo libre & 2,40 & 4,44 & 2,09 & 1,89 & 0,47 \\
Familiar & 1,45 & 1,69 & 0,25 & 0,82 & $4,63 * * *$ \\
Relación de pareja & 1,30 & 1,75 & 0,27 & 0,92 & $3,55 * * *$ \\
Relación sexual & 2,30 & 1,76 & 1,64 & 1,69 & 1,87 \\
Global & 2,60 & 1,35 & 2,93 & 1,07 & $-1,39$ \\
\hline
\end{tabular}

$* * * p<0,05, * * p<0,01, * * * p<0,001$. 
manifestaciones emocionales entre las que se encuentra la ansiedad y la depresión. La muestra de pacientes con VIH que nosotros hemos evaluado es representativa de los enfermos seropositivos en cuanto a sintomatología depresiva moderada coincidiendo con los resultados de los estudios de Perry y Fishman (1993) realizados con población bisexual y homosexual, los de Comer, Henker, Kemeny y Wyatt (2000) y Jones y Beach (2001) llevados a cabo con mujeres, y el de Davis, Metzger, Meyers, McLellan, Mulvaney, Navaline y Woody (1995) con usuarios seropositivos de drogas por vía parenteral. Algunos trabajos como el de Perkins, Stern, Golden, Murphy, Naftolowitz y Evans (1994) señalan que los mayores niveles de problemas emocionales como la ansiedad o la depresión encontrados con pacientes seropositivos en realidad no son consecuencia del impacto de la enfermedad, sino que suelen estar presentes ya antes del diagnóstico (especialmente en poblaciones como homosexuales o drogodependientes) y que por ello las diferencias solo aparecen cuando se comparan con la población general pero no cuando se compara con sujetos pertenecientes a estos colectivos que no se encuentran infectados por el VIH. No obstante, los datos obtenidos por Brown, Rundell, McManis, Kendall, Zachary y Temoshok (1992) al evaluar el estado mental de 442 varones después de conocer su estado serológico positivo al VIH les llevan a concluir que, estos pacientes tendrían más probabilidad que los restantes varones de su comunidad con similar perfil sociodemográfico, de padecer depresión mayor, trastornos de ansiedad e inhibición del deseo sexual. También Morrison et al. (2002) confirmaron que la prevalencia de depresión mayor era cuatro veces mayor en mujeres con infección por VIH que en mujeres seronegativas y aunque no habia diferencias estadísticamente significativas respecto al diagnóstico de trastornos de ansiedad, sí las había en el grado de la misma.

Otros trabajos como el de Thompson, Nanni y Levine (1996) incluyen los factores estresantes de los afectados por el VIH a la hora de evaluar su estado psicológico y comportamientos de riesgo. Sus resultados indican que los 105 varones con VIH que conforman su muestra presentaban niveles de depresión y percepción de estrés por encima de la población general e incluso superiores a individuos clínicamente deprimidos. Se obtenían correlaciones significativamente positivas entre estrés, factores estresantes (relaciones personales proble- máticas con la pareja o familiares, problemas financieros y enfermedad grave de algún amigo) y conductas de riesgo (mayor consumo de alcohol, tabaco y práctica sexual de riesgo). También Kelly, Murphy, Bahr y Koob (1993) hallaron una relación significativa entre el grado de depresión y el bajo apoyo social percibido, el locus externo de control sobre la salud y un mayor número de síntomas relacionados con la enfermedad.

Más recientemente y en nuestro contexto, Remor et al. (2001) encontraron que si bien la ansiedad es una reacción frecuente en el paciente con VIH, sus niveles probablemente cambien según la etapa de la enfermedad en la que éste se encuentre y que esta ansiedad parece depender en buena medida de la percepción de control que el paciente tenga sobre los acontecimientos estresantes vinculados a su enfermedad. De hecho, según Carrobles, Remor y Rodríguez-Almazora (2003), las estrategias de afrontamiento específicas del paciente pueden llegar explicar el $29 \%$ de la varianza de su distrés emocional. En la misma línea Martín et al. (2002) en su estudio realizado con pacientes VIH avanzados han encontrado una elevada prevalencia de ansiedad (77\%) y depresión (87\%) que alcanza criterios de gravedad en el $75 \%$ y $25 \%$ respectivamente.

Las investigaciones que conocemos sobre la autoestima de las personas afectadas por VIH son escasas. A pesar de ello, las conclusiones alcanzadas por algunos trabajos que abordan esta área junto a otros aspectos psicológicos (miedo a perder el atractivo físico e hipocondría) vienen a confirmar la baja competencia percibida y la escasa valoración que hacen los pacientes seropositivos de sí mismos (Morin et al., 1984).

Lo mismo cabe decir de estudios que investiguen el perfil de conducta de enfermedad de los pacientes con VIH. Por ello, nos remitimos a los resultados que presentan los enfermos oncológicos en su patrón de conducta de enfermedad y los datos de la muestra de pacientes con crisis de angustia, otros problemas de ansiedad y grupo control del estudio de Ballester y Botella (1993) donde también se aplicó el Illness Behaviour Questionaire. En la Figura 1 presentamos el perfil de conducta de enfermedad de pacientes seropositivos, oncológicos, con crisis de angustia, con otros problemas de ansiedad y población general. Como puede comprobarse, el perfil de conducta de enfermedad de los afectados por el VIH guar- 
Figura 1. Perfil de conducta de enfermedad en distintas poblaciones (puntuaciones medias)

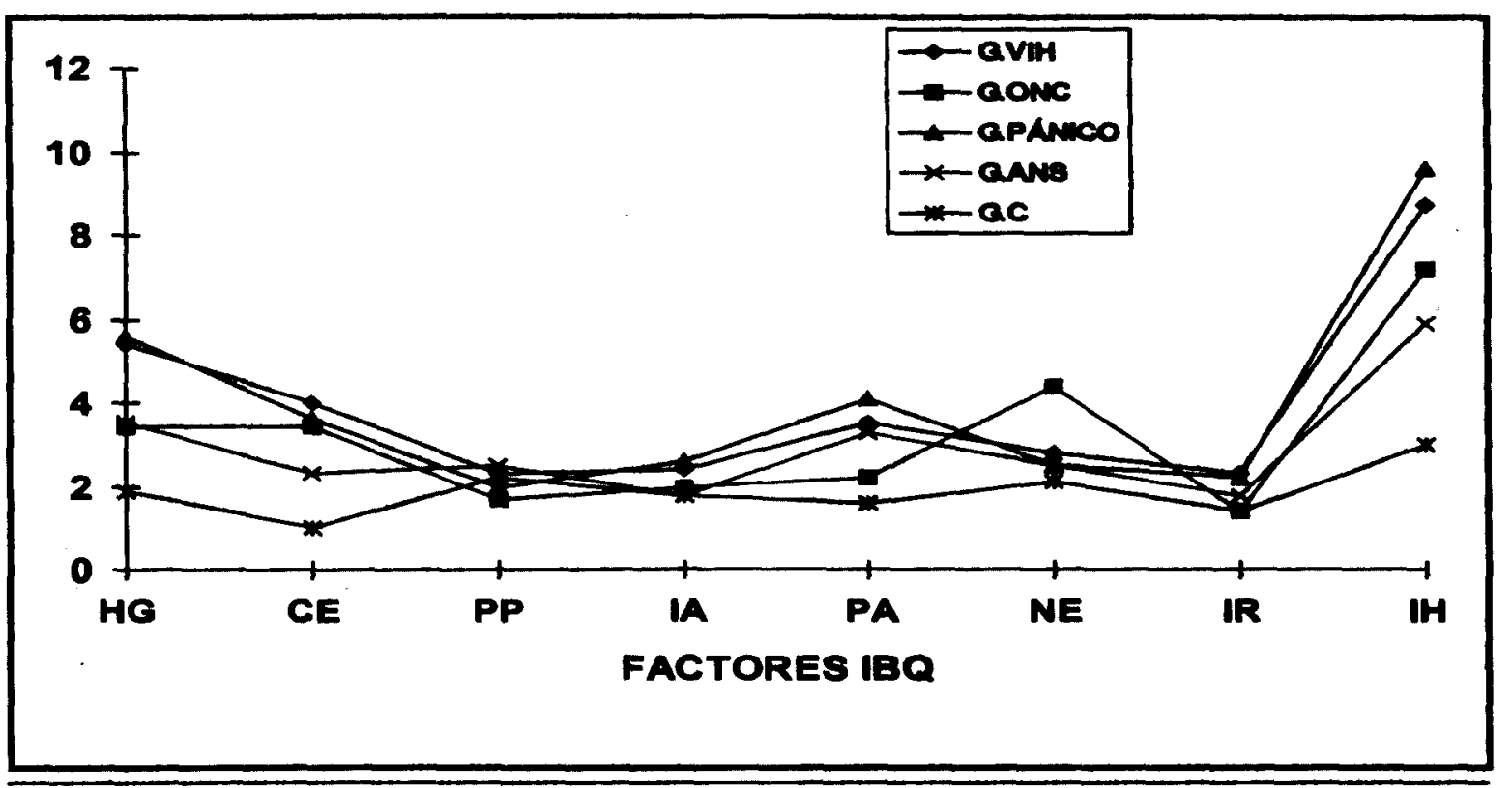

Nota: $\mathrm{HG}=$ Hipocondria General; $\mathrm{CE}=$ Convicción de estar Enfermo y Preocupación por los Síntomas; $\mathrm{PP}=$ Percepción Psicológica versus Somática de la Enfermedad; IA = Inhibición; PA = Perturbación Afectiva; NE = Negación de Problemas Cotidianos; IR = Irritabilidad o Enfado y Fobia a la Enfermedad; IH = Indice Whiteley de Hipocondria.

da similitudes con el de los pacientes con crisis de pánico. Ambos colectivos de pacientes coinciden en su alto grado de hipocondría, tanto en el factor Hipocondría General como en el Indice de Whiteley de Hipocondría. Si nos centramos en el papel de la hipocondría de los portadores del VIH, el estudio de Tsao, Dobalian, Moreau y Dobalian (2004) concluye que la disminución de los sintomas fisicos de la enfermedad y la percepción de los mismos es primordial para la salud mental de los afectados. Esta recomendación que, en principio, puede ser beneficiosa para el bienestar de las personas con VIH al permitir una rápida intervención puede, sin embargo, desembocar, según Garcia-Huete (1993), en estados de hipervigilancia, comportamientos de auto-observación y evitación social que generan ansiedad y desgastan el sistema inmunitario. Tanto los pacientes con VIH como los pacientes con crisis de angustia presentan niveles significativamente más elevados en hipocondría y número de problemas afectivos con respecto a los enfermos de cáncer, ansiedad y población general.

Dos son las características definitorias del perfil de conducta de enfermedad peculiar de los enfermos por VIH. Por un lado, su elevada irritabilidad ligeramente superior a la de los pacientes con crisis de pánico y significativamente superior a la irritabilidad de los enfermos de cáncer y el grupo control. Por otro lado, su menor, pero estadísticamente significativa, tendencia a negar los problemas cotidianos o explicarlos a partir de sus problemas fisicos en comparación con los pacientes oncológicos.

De todos modos, quizás la conducta de enfermedad de los pacientes puede verse influida por otros factores como puede ser el tipo de colectivo estudiado dentro de la población de seropositivos. Así, en un estudio realizado en el año 1992 por Lazzari, Campione y Chiodo (1992) en el que se comparaba la conducta de enfermedad de pacientes seropositivos drogodependientes, homosexuales y heterosexuales, se encontró que los drogodependientes presentaban una menor negación, mayor irritabilidad y una percepción más psicológica del problema que los otros grupos. Por otra parte, sabemos que variables como un bajo grado de depresión, la capacidad para expresar las emociones y niveles adecuados de apoyo social tienden a conducir al paciente hacia una conducta de enfermedad más adaptativa (Grassi, Righi, Makoui, Sighinolfi, Ferri y Ghinelli, 1999). 
En cuanto a la percepción de apoyo social de nuestros afectados por el VIH, el estudio longitudinal realizado por Burgoyne (2005) durante 4 años con 34 pacientes con VIH destaca la importancia de la percepción del apoyo social para estos enfermos como factor asociado a la adherencia a los tratamientos médicos, aspecto éste fundamental para la salud de los pacientes (Ballester, 2002). Así, confirma las observaciones empíricas y descriptivas que resaltan el peso fundamental del entorno del enfermo para su mejoría física y mental. La carencia de apoyo social junto a otras circunstancias (bajo nivel educativo, antecedentes psiquiátricos personales y/o familiares, percepción de no control de la situación etc.) aumenta significativamente la aparición de sintomatología psicopatológica. Las conclusiones alcanzadas por Theorell, Blomkvist, Jonsson, Schulman, Berntorp y Stigendal (1995) en su estudio longitudinal con pacientes hemofilicos positivos al VIH complementan los anteriores hallazgos sobre apoyo social. Estos autores informan de la influencia negativa que ejerce un limitado apoyo emocional en el estado inmunológico de estos pacientes. En este sentido, en los pacientes con cáncer también se ha demostrado la influencia de las relaciones interpersonales en el estado inmunitario. Los enfermos oncológicos con un fuerte apoyo social tienen asociado un mejor pronóstico (Levy, Herberman, Maluish, Schliew y Lippman, 1985) y mayor supervivencia (Funch y Marshall, 1983; Weisman y Worden, 1975). Por otra parte, Ayuso (1997) afirma que las posibilidades de conseguir redes de apoyo social son mayores entre los pacientes VIH hemofilicos que entre los UDVP (usuarios de drogas por vía parenteral). Según Eakin y Taylor (1990), quienes abordaron el aspecto psicosocial del SIDA en los trabajadores sanitarios, se pueden identificar tres modelos de respuesta: evitación, compromiso y adaptación. La evitación, motivada por miedos irracionales, prejuicios e incapacidades, interfiere en la no normalización de esta enfermedad como cualquier otra. Estas cuestiones bien pueden ser generalizables al ámbito social del paciente seropositivo, y explicar así, o al menos en parte, la disminución significativa de apoyo social que reciben los enfermos de VIH en comparación a la atención social hacia los enfermos oncológicos.

En nuestra opinión, el conocimiento del estado emocional y la conducta de enfermedad de los pacientes infectados por VIH puede ser de gran utilidad para desarrollar programas de intervención psicológica que, con el fin de aumentar el bienestar emocional de los pacientes, su afrontamiento de la enfermedad o mejorar su adherencia a los tratamientos médicos, estén completamente ajustados al perfil de estos pacientes. Sin embargo, somos conscientes de algunas limitaciones y aspectos de nuestra investigación que podrían ser mejorados en el futuro como son: la inclusión de la carga viral como marcador biológico del estado clínico del paciente con VIH, la aplicación de instrumentos de evaluación concretos que discriminen las variables médicas que pueden estar influyendo en su estado emocional, ampliar el tamaño de la muestra, controlar el nivel de gravedad o avance de la enfermedad de los pacientes así como la interferencia de los tratamientos farmacológicos y de sustancias psicoactivas y/o programas de metadona de los ADVP seropositivos. También, sería interesante diferenciar en futuros estudios entre los trastornos mentales de los pacientes previos a la infección o posteriores al conocimiento de su estado positivo al VIH.

\section{REFERENCIAS}

Ayers, M., Abrams, D., Newell, T., y Friedrich, F. (1989). Performance of individuals with AIDS on the LuriaNebraska Neuropsychological Battery. International Journal of Clinical Neuropsychology, 9, 101-105.

Ayuso, J.L. (1997). Trastornos neuropsiquiátricos en el SIDA. Madrid: McGraw Hill Interamericana.

Ballester, R. (1999). Prevención y tratamiento Psicológico del VIH/SIDA. Madrid: UNED-Fundación Universidad Empresa.

Ballester, R. (2002). Adhesión terapéutica: revisión histórica y estado de la cuestión en la infección por VIH/SIDA. Revista de Psicopatologia y Psicologia Clínica, 7, 151-175.

Ballester, R. (2005). Aportaciones desde la Psicologia al tratamiento de las personas con Infección por VIH. Revista de Psicopatología y Psicologia Clínica (número monográfico), 10, 53-69.

Ballester, R., y Botella, C. (1993). Autoconciencia y conciencia corporal en pacientes con trastorno con angustia. Análisis y Modificación de Conducta, 19. 613-642.

Ballester, R., y Botella, C. (1993). Perfil de conducta de enfermedad en pacientes con crisis de angustia. Análisis y Modificación de Conducta, 19, 233-265.

Ballesteros, R., y Carrobles, J.A. (1989). Evaluación versus tratamiento. Evaluación conductual: Metodologia y Aplicaciones. Madrid: Pirámide. 
Beck, A.T., y Steer, R.A. (1993). BDI: Beck Depression Inventory Manual. Nueva York: Psychological Corporation.

Bermúdez, J. (1978). Ansiedad y rendimiento. Revista de Psicologia General y Aplicada, 151,183-207.

Bor, R. (1991). Efficacy of psychological therapies for people with disease. AIDS Care, 4, 405-407.

Brown, G.R., Rundell, J.R., McManis, S.E., Kendall, S.N., Zachary, R., y Temoshok, L. (1992). Prevalence of psychiatric disorders in early stages of HIV infection. Psychosomatic Medicine, 54, 588-601.

Bruce, B.K., y Stevens, V. M. (1992). AIDS-related obsessive compulsive disorder: A treatment dilemma. Journal of Anxiety Disorders, 6, 79-88.

Byrgoyne, R. (2005). Exploring direction of causation between social support and clinical outcome for HIV-positive adults in the context of highly active antiretroviral therapy. AIDS Care, 17, 111-124.

Byrne, D.G., y Whyte, H. M. (1978). Dimensions of illness behavior in survivors of myocardial infarction. Journal of Psychosomatic Research, 22, 485-491.

Carrobles, J.A., Remor, E., y Rodriguez-Alzamora, L. (2003). Afrontamiento, apoyo social percibido y distrés emocional en pacientes con infección por VIH. Psicothema, 15, 420-426.

Comer, L.K., Henker, B., Kemeny, M., y Wyatt, G. (2000). Illness disclosure and mental health among women with HIV/AIDS. Journal of Community and Applied Social Psychology, 10, 449-464.

Davis, R.F., Metzger, D.S., Meyers, K., Mc Lellan, A.T., Mulvaney, F.D., Navaline, H.A., y Woody, G.E. (1995). Long-term changes in psychological symptomatology associated with HIV serostatus among male injecting drug users. AIDS, 9, 73-9.

Davis, S. (2004). Clinical sequelae affecting quality of life in the HIV-infected patient. Journal of the Association of Nurses in AIDS Care, 15, 28-33.

Eakin, J.M., y Taylor, K.M. (1990). The psychosocial impact of AIDS on health workers. AIDS, 4, 5257-5262.

Edo, M. (2003). Perfil psicosocial, estado emocional y conducta de enfermedad en pacientes con infección por VIH/SIDA. Universitat Jaume I de Castelló: Tesis Doctoral.

Edo, M.T., Ballester, R., y Pascual, L.M. (2003). Percepción de apoyo social en pacientes con Infección por VIH/SIDA y oncológicos. II Congreso Latinoamericano de Psicología de la Salud, p. 270. Cartagena de Indias.

Flores, E., Borda, M., y San Gregorio, A. (2006). SIDA y exclusión social: alteraciones emocionales. Revista de Psicopatologia y Psicología Clínica, 11, 37-49.

Funch, D.P., y Marshall, J. (1983). The role of stress, social support and age in survival from breast cancer. Journal Psychosomatic Research, 27, 77-83.

Garcia-Huete, E. (1993). SIDA: Apoyos en el entorno personal, familiar y laboral. Madrid: Eudema.
Goldsmith, R.E. (1986). Dimensionality of the Rosenberg Self-Esteem Scale. Journal of Social Behavior and Personality, 1, 253-264.

Grassi, L., Righi, R., Makoui, S., Sighinolfi, L., Ferri, S., y Ghinelli, F. (1999). Illness Behavior, emotional stress and psychosocial factors among asymptomatic HIVinfected patients. Psychotherapy and Psychosomatics, 68, 31-38.

Green, G. (1994). Positive sex: sexual relationships following an HIV-positive diagnosis. En P.Aggleton, P. Davies y G. Hart: AIDS: foundations for the future (pp.136-146). Londres: Taylor \& Francis.

Hunter, J. (1993). Mental health aspects of HIV infection. Can-Fam-Physician, 39, 1415-1424.

Jones, D.J., y Beach, S.R.(2001). Disease status in african single mothers with HIV: The role of depressive symptons. Health Psychology, 20, 417-423.

Kelly, J., Murphy, D., Bahr, R., y Koob, J. (1993). Factors associated with severity of depression and high-risk sexual behavior in patients diagnosed with HIV Infection. Health Psychology, 12, 215-219.

Kermani, E.J., Borod, J.C., Brown, P.H. y Tunnell, G. (1985). New psychopathologic findings in AIDS: Case report. Journal of Clinical Psychiatry, 46, 240-241.

Kim, C.R., y Rickman, L.S. (1988). Psychological aspects of the acquired immunodeficiency syndrome: A case report and review of the literature. Military Medicine, 153, 638-641.

King, M.B. (1989). Psychosocial status of 192 out-patients with HIV infection and AIDS. British Journal of Psychiatry, 154, 237-242.

Krikorian, R., Kay, J., y Liang, W, M. (1995). Emotional distress, coping and adjustment in human immunodeficiency virus infection and acquired immune deficiency syndrome. Journal of Nervous and Mental Disease, 183, 293-298.

Lazzari, C., Campione, F., y Chiodo, F. (1992). Illness behavior in HIV Ab+ve subjects. $8^{\text {th }}$ International AIDS Conference. Amsterdam, 19-24 Julio. Abstract 7299.

Levenson, A.I. (1988). Psychiatric aspects of AIDS. 55th Annual Meeting of the National Association of Private Psychiatric Hospitals (1988, Phoenix, Arizona). Psychiatric Hospital, 19,109-113.

Levy, S., Herberman, R., Maluish, A., Schliew, B., y Lippman, M. (1985). Prognostic risk assessment in primary breast cancer by behavioral and immunological parameters. Health Psychology, 4, 99-113.

Martín, I., Cano, R., Pérez, P., Aguayo, M., Cuesta, F., Rodríguez, P., y Pujol, E. (2002). Calidad de vida, aspectos psicológicos y sociales en pacientes con Infección por VIH avanzada. Anales de Medicina Interna, 19, 396-404.

Martin, J.L., y Dean, L. (1993). Effects of AIDS-related bereavement and HIV related illness on psychological distress among gay men: a 7-year longitudinal study, 
1985-1991. Journal of Consulting and Clinical Psychology, 61, 94-103.

Morin, S.F., Charles, K.A., y Malyon, A.K. (1984) The psychological impact of AIDS on gay men. American Psychologist, 39, 1288-1293.

Morrison, M., Petitto, J., Ten Have, T., Gettes, D., Chiappini, M., Weber, A., Brinker-Spence, P., Bauer, R., Douglas, S., y Evans, D. (2002). Depressive and anxiety disorders in women with HIV Infection. American Journal of Psychiatry, 159, 789-796.

Naber, D., Perro, C., Schick, U., Sadri, I., et al., (1989). Psychiatrische Symptome und neuropsychologische Auffalligkeiten bei HIV-Infizierten/ Psychiatric symptoms and neuropsychological abnormalities in HIV infection. Nermenarzt, 60, 80-85.

Nichols, S.E. (1983). Psychiatric aspects of AIDS. Psychosomatics, 24, 1083-1089.

Pace, J., Brown, G.R., Rundell, J.R., Paolucci, S., et al. (1990). Prevalence of psychiatric disorders in a mandatory screening program for infection with human immunodeficiency virus: A pilot study. Military Medicine, 155, 76-80.

Perkins, D., Stern, R., Golden, R., Murphy, C., Naftolowitz, D., y Evans, D. (1994). Mood disorders in HIV Infection: prevalence and risk factors in a nonepicenter of the AIDS epidemic. American Journal of Psychiatry, 151, 233-236

Perry, S.W., y Fishman, B. (1993). Depression and HIV: How does one affect the other? Journal of the American Medical Association, 270, 2609-2610.

Pilowsky, I., y Spence, N. (1983). Manual for the Mllnes Behaviour Questionnaire. University of Adelaide.

Piña, J.A., y Robles, S. (2005). Psicología y VIH/SIDA en México: su prevención como base en un modelo psicológico de salud biológica. Revista de Psicopatologia y Psicologia Clinica, 10, 71-84.
Remor, E., Carrobles, J.A., Arranz, P., Donate, A.M., y Ulla, S. (2001). Ansiedad y percepción de control en la infección por VIH y SIDA. Psicologia Conductual, 9, 323-336.

Rosenberg, M. (1979). Conceiving the self. New York: Basic Books.

Ross, M.W., y Rosser, B.S. (1988). Psychological issues in AIDS-related syndromes. Patient Education and Counseling, 11,17-28.

Spielberger, C.D., Gorsuch, R.L., y Lushene, R. E.(1982). Cuestionario de Ansiedad Estado-Rasgo (STAI) Manual. Madrid: TEA.

Theorell, T., Blomkvist, V., Jonsson, H., Schulman, S., Berntorp, E. y Stigendal, L. (1995). Social support and the development of immune function in human immunodeficiency virus infection. Psychosomatic Medicine, 57, 32-36.

Thompson, S., Nanni, C., y Levine, A. (1996). The stressors and stress of being HIV-positive. AIDS Care, 8, 514.

Tsao, J., Dobalian, A., Moreau, C., y Dobalian, K.(2004). Stability of anxiety and depression in a national sample of adults with human immunodeficiency virus. Journal of Nervous and Mental Disease, 192, 111-118.

Ward, R.A. (1977). Aging group consciousness. Implications in an older sample. Sociology and Social Research, 61, 496-519.

Weinrich, J.D., Atkinson, J.H., McCutchan, J.A., y Grant, I. (1995). Is gender dysphoria dysphoric? Elevated depression and anxiety in gender dysphoric and nondysphoric homosexual and bisexual men in an HIV sample. HNRC Group. Archives of Sexual Behavior, 24, 55-72.

Zonderman, A.B., Heft, M.W., y Costa, P.T: (1985). Does the Illness Behavior Questionnaire measure abnormal illnesss behavior? Health Psychology, 4, 425-429. 